\title{
Comparison of Endovenous Laser and Radiofrequency Ablation in Treating Varices in the Same Patient
}

\author{
Orhan Bozoglan ${ }^{1 *}$, Bulent Mese ${ }^{1}$, Erdinc Eroglu' ${ }^{1}$, Hasan Cetin Ekerbiçer², Alptekin Yasim¹ \\ ${ }^{1}$ Kahramanmaras Sutcu Imam University, Faculty of Medicine, Departments of Cardiovascular Surgery, Turkey \\ ${ }^{2}$ Sakarya University, Faculty of Medicine, Departments of Public Health, Turkey
}

\author{
*Correspondence to \\ Orhan Bozoglan, MD; \\ Kahramanmaras Sutcu Imam \\ University Faculty of Medicine, \\ Department of Cardiovascular \\ Surgery, Kahramanmaras, Turkey. \\ Tel: +90- 5323239776 ; \\ Fax: +90-344 2803409; \\ Email: \\ orhanbozoglan1975@hotmail.com
}

Published online 8 January 2017

\begin{abstract}
Introduction: To compare endovenous laser ablation (EVLA) and radiofrequency ablation (RFA) in different legs in the same individual in patients with venous insufficiency.

Methods: Sixty patients with bilateral saphenous vein insufficiency were included. EVLA or RFA was applied to one of the patient's legs and RFA or EVLA to the other leg.

Results: EVLA and RFA complications were hyperemia at $20.7 \%$ and $31.0 \%$, ecchymosis at $31.0 \%$ and $51.7 \%$ and edema at $27.6 \%$ and $65.5 \%$, respectively. The rate of recanalization was $6.8 \%$ in the RFA group. No recanalization was observed in EVLA group. The level of patients satisfied with EVLA was $51.7 \%$, compared to $31.0 \%$ for RFA, while $17.2 \%$ of patients were satisfied with both procedures. Times to return to daily activity were 0.9 days in the EVLA group and 1.3 days in the RFA group.

Conclusion: EVLA procedure may be superior to RFA in certain respects.

Keywords: Varicose veins; Endovascular laser ablation; Radiofrequency ablation.
\end{abstract}

\section{Introduction}

Ligation and stripping was for years the most frequently employed therapeutic option in the treatment of great saphenous vein insufficiency. However, in association with technological advances, there has been continual research into treating the disease using endovenous methods. Research into sclerosing the venous wall using thermal methods in particular has recorded considerable progress, and in 2001 Navarro et al published the first application of thermal endovenous ablation using an 810 $\mathrm{nm}$ diode laser. ${ }^{1}$ Since then, there has been increasing progress in laser technology, and numerous studies have been performed using different wavelengths and types of laser. $^{2-7}$ The Food and Drug Administration (FDA)-approved lasers today are $810,940,980$ and $1470 \mathrm{~nm}$ diode lasers and 1319 and $1320 \mathrm{~nm}$ neodymium-doped yttrium aluminium garnet (Nd:YAG) lasers. In parallel to advances in laser technology, studies began being performed concerning thermal ablation of the saphenous vein using radiofrequency energy, and permission for the use of radiofrequency energy in endovenous ablation was granted by the FDA in 1999. In 2002, Weiss and Weiss reported the first patients receiving thermal ablation using radiofrequency energy. ${ }^{8}$ Numerous studies using radiofrequency ablation (RFA) were subsequently published. ${ }^{9-11}$ Studies comparing endovenous laser ablation (EVLA) and RFA then began being published. These studies generally reported equal success between EVLA and RFA, albeit with fewer side-effects and greater patient satisfaction with RFA. ${ }^{12,13}$ However, all these studies compared laser energy at low wavelengths $(810,940$ and $980 \mathrm{~nm})$ with radiofrequency. However, high wavelength laser energy and radial fiber have been shown to produce better patient satisfaction and fewer side-effects compared to low wavelength laser energy and bare fiber. ${ }^{14}$ There are no clinical studies in the literature comparing laser energy at a wavelength of 1470 or more with RFA. We therefore planned this study in order to assess patients receiving EVLA with laser energy at a wavelength of $1470 \mathrm{~nm}$ and radial fiber and patients receiving RFA in terms of procedure success, complications and patient satisfaction. In order to eliminate patient-related variables, EVLA was applied to one leg of patients with bilateral saphenous vein insufficiency and RFA to the other leg.

\section{Methods}

Sixty patients, 28 men and 32 women, with symptomatic great saphenous vein insufficiency in both lower extremities and presenting to the cardiovascular surgery clinic between January and December 2013, were enrolled. Patients' ages ranged between 29 and 64 (mean $42.8 \pm 10.0$ years). Sixty EVLA and 60 RFA procedures were applied to the saphenous veins in the lower extremities of the 60 patients. Ethical committee approval was obtained before 
the study began. Patients with unilateral vena saphena magna (VSM) insufficiency, patients receiving the same technique to both legs, patients not permitting intervention on both legs in different sessions and patients who permitted interventions in both legs in separate sessions but in whom technical failure occurred in one or both sessions were excluded. Patients were classified on the basis of CEAP (clinical severity, etiology, anatomy and pathophysiology) before the procedure began. Venous clinical severity score (VCSS) values based on scoring of pre-procedural clinical symptoms and findings were recorded. EVLA and RFA procedures were decided on in the light of insufficiency in both existing VSM at colored Doppler ultrasonography (CDUSG) performed for diagnostic purposes. No advanced insufficiency or obstruction was determined in the deep veins of any extremity. A $12 \mathrm{~W}$ diode laser source with a wavelength of $1470 \mathrm{~nm}$ (Biolas-15D, Del YCHI GMBH, Duisburg, Germany) and radial fiber (EVLAS Circular-2, FG Group, Ankara, Turkey) were used for EVLA and an $\mathrm{EVRF}^{\circledR}$ : endovenous radio frequency CR45i device and catheter (F-Care Systems NV, Antwerp, Belgium) were used for RFA. Percutaneous entry was performed with a $21 \mathrm{G}$ needle accompanied by caudal section ultrasonography (USG) appropriate for treatment of saphenous vein with reflux determined in all patients under local anesthesia. Tumescent local anesthesia consisting of $20 \mathrm{~mL} 2 \%$ prilocaine, $500 \mathrm{~mL} 0.9 \%$ isotonic solution $\left(+4^{\circ} \mathrm{C}\right), 20 \mathrm{~mL} 8.4 \%$ sodium bicarbonate and $0.5 \mathrm{mg}$ adrenalin was administered to the area surrounding the saphenous vein with 19-21G needles guided by USG.

Endovenous Laser Ablation Procedure

Laser energy was applied by adjusting the laser parameters $(12 \mathrm{~W}, 1.2-1.8 \mathrm{~mm} / \mathrm{s}$ withdrawal speed) in pulse mode ( 0.2 second interval) depending on the vein diameter and depth from the skin of the saphenous vein, such as to be greater in those areas close to the saphenofemoral junction (SFJ).

Radiofrequency Ablation Procedure

Radiofrequency energy was applied to the saphenous vein in the form of $25 \mathrm{~W}$ every $0.5 \mathrm{~cm}$ from the distal aspect of the SFJ $(50 \mathrm{~W} / \mathrm{cm})$. Analgesic (paracetamol) was prescribed for all patients after both procedures.

Pain during and after the procedure was assessed using a visual analog scale (VAS). Patients indicated the pain felt on a scale of 1 to 5. Patients' analgesic requirements were recorded. An elastic bandage was applied for 2 days to the leg receiving the procedure. Compression socks were subsequently recommended for 3 months. Patients were encouraged to return to their daily activities as early as possible. Times to return to daily activities were recorded. Follow-ups were performed clinically on the second day post-procedure and both clinically and using CDUSG on the 1st week and at the first, third and sixth months. Saphenous vein occlusion, recanalization, perforating veins and residual varicosities were recorded by CDUSG. Major and minor complications were investigated. No pake excision was performed on any patient during EVLA or RFA.

\section{Statistical Analysis}

Data were expressed as mean \pm standard deviation (SD) or as median and range. Demographic and clinical measures were tested using paired samples $t$ tests for parametric variables and Wilcoxon signed ranks tests for non-normally distributed data. McNemar test was used to analyze quantitative data. All calculations were performed using SPSS version 17.0 (SPSS Inc., Chicago, IL, USA). $P<0.05$ was considered statistically significant.

\section{Results}

All patients had primary etiology, and pathophysiology was associated with reflux in the entire extremity. All patients receiving EVLA and RFA were symptomatic in both legs. No statistically significant difference was determined between legs on the basis of CEAP and VCSS classification at preoperative assessment. Mean duration of reflux in the SFJ was 3.4 seconds in the EVLA group and 3.8 seconds in the RFA group. EVLA and RFA procedures were performed on 120 saphenous veins. Mean diameters of saphenous veins receiving EVLA were $9.6 \mathrm{~mm}$ at the level of the SFJ and $8.2 \mathrm{~mm}$ at knee level. The equivalent values in patients receiving RFA were $10.3 \mathrm{~mm}$ and 8.4 $\mathrm{mm}$, respectively. Length of saphenous vein undergoing procedure was $27.4 \mathrm{~cm}$ in subjects receiving EVLA and $26.5 \mathrm{~cm}$ in those receiving RFA. Depth of saphenous vein from the skin was $15.3 \mathrm{~mm}$ in the EVLA group and 14.7 $\mathrm{mm}$ in the RFA group. Duration of procedures was 31.2 minutes for EVLA and 32.7 minutes for RFA. No significant difference was determined between the groups. Demographic and clinical findings are shown in detail in Table 1. Preoperative pain score on VAS was 1.4 in the EVLA group and 1.7 in the RFA group, although the difference was not significant. Postoperative pain score was 1.2 in the EVLA group and 1.4 in the RFA group. The difference was statistically significant $(P<0.035)$. Postoperative analgesic requirement was 1.7 units/day in the EVLA group and 1.9 units/day in the RFA group. The difference was not significant. Length of time to start postoperative activity was 0.9 days in the EVLA group and 1.3 days in the RFA group, and the difference was again significant $(P<0.001)$. Time to return to work was 1.8 days in the EVLA group and 2.1 days in the RFA group, and the difference was not significant. Postoperative data are shown in Table 2. Postoperative minor complications were determined in the form of induration, ecchymosis and edema. Induration developed in $69.0 \%$ of patients in the EVLA group and $79.3 \%$ of those in the RFA group. The difference was not significant. Ecchymosis developed in 31.0\% of the patients in the EVLA group and in $51.7 \%$ of those in the RFA group. The difference was not significant. Edema developed in $27.6 \%$ of the patients in the EVLA group and $65.5 \%$ of those in the RFA group. The difference was statistically significant $(P<0.007)$. Induration, ecchymosis and edema resolved entirely at the end of 2 weeks. No 
Table 1. Demographic and Clinical Data

\begin{tabular}{lccc}
\hline & EVLA $(\mathbf{n}=\mathbf{6 0})$ & RFA $(\mathbf{n = 6 0})$ & $\boldsymbol{P}$ \\
\hline VCSS & $9.7 \pm 2.5$ & $9.9 \pm 2.5$ & 0.196 \\
CEAP & $3.2 \pm 0.4$ & $3.2 \pm 0.4$ & 1.000 \\
VSM diameter (SFJ) $\mathrm{mm}$ & $9.6 \pm 1.7$ & $10.3 \pm 2.8$ & 0.213 \\
VSM diameter (knee) mm & $8.2 \pm 1.4$ & $8.4 \pm 2.3$ & 0.633 \\
Mean SFJ reflux time (s) & $3.4 \pm 1.4$ & $3.8 \pm 1.3$ & 0.102 \\
Distance from skin & $15.3 \pm 7.3$ & $14.5 \pm 7.3$ & 0.212 \\
Length of saphenous vein & $27.4 \pm 3.4$ & $26.5 \pm 6.5$ & 0.108 \\
Duration of procedure & $31.2 \pm 4.7$ & $32.7 \pm 6.5$ & 0.205 \\
\hline
\end{tabular}

Abbreviations: EVLA, endovenous laser ablation; RFA radiofrequency ablation; VCSS, venous clinical severity score; CEAP, clinical severity, etiology, anatomy and pathophysiology; VSM, vena saphena magna; SFJ, saphenofemoral junction.

Table 2. Postoperative Data

\begin{tabular}{lccc}
\hline & EVLA $(\mathbf{n = 6 0})$ & RFA $(\mathbf{n = 6 0})$ & $\boldsymbol{P}$ \\
\hline Pain (intraoperative)/day & $1.4 \pm 0.6$ & $1.7 \pm 0.8$ & 0.074 \\
Pain (postoperative)/day & $1.2 \pm 0.4$ & $1.4 \pm 0.5$ & $0.035^{*}$ \\
Analgesic requirement & $1.7 \pm 0.6$ & $1.9 \pm 0.4$ & 0.063 \\
Time to return to activity & $0.9 \pm 0.8$ & $1.3 \pm 1.1$ & $0.001^{*}$ \\
Time to return to work & $1.8 \pm 0.8$ & $2.1 \pm 1.2$ & 0.549 \\
\hline
\end{tabular}

Abbreviations: EVLA, endovenous laser ablation; RFA, radiofrequency ablation.

Table 3. Complications After Endovenous Laser Therapy and Radiofrequency Ablation

\begin{tabular}{lccc}
\hline & EVLA $(\mathbf{n = 6 0})$ & RFA $(\mathbf{n = 6 0})$ & $\boldsymbol{P}$ \\
\hline Induration & $20.7 \%$ & $31.0 \%$ & 0.508 \\
Ecchymosis & $31.0 \%$ & $27.6 \%$ & 0.146 \\
Edema & $27.6 \%$ & $65.5 \%$ & $0.007^{*}$ \\
Paresthesia & 0.0 & 0.0 & - \\
Deep vein thrombosis & 0.0 & 0.0 & - \\
Pulmonary embolism & 0.0 & 0.0 & - \\
\hline
\end{tabular}

Abbreviations: EVLA, endovenous laser ablation; RFA, radiofrequency ablation.

major complication (such as DVT, pulmonary embolism or skin burn) was observed in any patient. Complications after endovenous laser therapy and RFA are shown in Table 3. When asked once both procedures had been performed which they were more satisfied with, $31 \%$ of patients responded RFA, 51.7\% EVLA and 17.2\% both. Recanalization developed in 4 saphenous veins in the RFA group during monitoring, a recanalization rate of $6.8 \%$. Complete occlusion was determined in 60 (100\%) saphenous veins at sixth month follow-up after EVLA in the EVLA group.

\section{Discussion}

Chronic venous insufficiency (CVI) and lower extremity varices that develop in association with this are an important clinical condition that significantly affect quality of life and have socioeconomic consequences. ${ }^{15}$ Significant progress has been made in the treatment of varicose veins in the last 10 years. Endovenous ablation techniques have to a large extent replaced surgery. Thermal endovenous procedures such as RFA and EVLA have become the most commonly used techniques. Several studies have compared these two different forms of ablation. There is no general consensus on which method is superior. Publications generally report that both methods have the same levels of successful ablation, but that pain levels and rates of complications following RFA are lower compared to EVLA. ${ }^{12,16-20}$ However, these studies have generally used low wavelength laser. Several recent studies suggest that better results are achieved with higher laser wavelengths. One recent study compared radial laser fiber at a wavelength of $1470 \mathrm{~nm}$ and bare fiber at a wavelength of 980 $\mathrm{nm}$ and reported better patient comfort with $1470 \mathrm{~nm}$ radial fiber. ${ }^{14}$ Therefore, if we really wish to compare the two methods, we need to compare $1470 \mathrm{~nm}$ radial laser and RFA. The most important factor determining patient comfort is sensation of pain. This may vary from person to person, and when the two techniques are compared in different patients it may be impossible to make a truly objective assessment due to variations in different patients' pain thresholds. Our purpose was therefore to compare the effectiveness and side-effects of these methods by applying the two different ablation techniques to different legs in the same patient. No previous studies have compared these two techniques applied to different extremities in the same patient. We determined ablation rates of $100 \%$ in the EVLA group and $93.2 \%$ in the RFA group. The difference was not statistically significant, however. In their meta-analysis, van den Bos et al evaluated 119 studies and determined success rates of $94 \%$ for EVLA and $84 \%$ for RF on the basis of results for 12320 legs. ${ }^{21}$ Almeida et al reported recanalization rates of 5.5\% for RF and $1.7 \%$ for EVLA. ${ }^{16}$ Puggioni et al reported success rates at 1-month follow-up of $100 \%$ for EVLA and $96 \%$ for RF. ${ }^{17}$ This is because in performing ablation with RF the catheter has to touch the vein wall, while thermal ablation is possible without laser energy making contact. All previous studies have reported that the greatest superiority of RF over EVLA is greater patient satisfaction..$^{12,17-19}$ In our study, however, patient satisfaction was higher in the EVLA group, while better results for criteria such as intraoperative and postoperative pain, postoperative analgesic requirement, return to activity and return to work were obtained in the EVLA group. Of these, postoperative pain and time to return to activity were statistically significant $(P<0.035$ and $P<0.001$, respectively). We attribute this both to the low wavelengths in previous studies and to the use of bare tip laser catheters. Since high wavelength laser rays use water as a chromophore they are better able to penetrate the vein wall. In addition, the radial dissemination of rays permits a more homogeneous contact with the vein wall and reduces the incidence of perforation. No procedure-related major complication (DVT, pulmonary embolism, skin burn) developed in this study. Minor complication levels were lower in the EVLA group. The point that should not be forgotten here is that the majority of minor complications (hematoma, ecchymosis) occur not in association with the procedure but with the application of tumescent anesthesia. Careful application of tumescent 
anesthesia will reduce these complications to a minimum. In that event, the most important difference between the two techniques will be sensation of pain and occlusion rate. Sensation of pain is lower in the EVLA group (with a $1470 \mathrm{~nm}$ wavelength and radial fiber).

\section{Conclusion}

Comparing the two techniques in the same patient in this study reduces patient-dependent factors to a minimum. Patient satisfaction can thus be assessed more objectively. In conclusion, EVLA and RFA have similar success rates. However, in terms of pain and patient satisfaction, EVLA at a wavelength of $1470 \mathrm{~nm}$ and using radial fiber is superior to RFA.

\section{Ethical Considerations}

The ethics committee of Kahramanmaraş Sütçü İmam University approved this study (REC number: 2013/14-05).

\section{Conflict of Interests}

Orhan Bozoglan and other co-authors have no conflict of interest.

\section{References}

1. Navarro L, Min RC, Bone C. Endovenous laser: a new minimally invasive method of treatment for varicose veins-preliminary observations using an $810 \mathrm{~nm}$ diode laser. Dermatol Surg. 2001;27:117-122. doi:10.1046/j.15244725.2001.00134.x.

2. Proebstle TM, Lehr HA, Kargl A, et al. Endovenous treatment of the greater saphenous vein with a $940-\mathrm{nm}$ diode laser: thrombotic occlusion after endoluminal thermal damage by laser-generated steam bubbles. J Vasc Surg. 2002;35:729-736. doi:10.1067/mva.2002.121132.

3. Yu DY, Chen HC, Chang SY, Hsiao YC, Chang CJ. Comparing the effectiveness of 1064 vs. $810 \mathrm{~nm}$ wavelength endovascular laser for chronic venous insufficiency (varicose veins). Laser Ther. 2013;22:247-253. doi:10.5978/ islsm.13-or-19.

4. Goldman MP, Mauricio M, Rao J. Intravascular 1320-nm laser closure of the great saphenous vein: a 6- to 12-month follow-up study. Dermatol Surg. 2004;30:1380-1385. doi:10.1111/j.1524-4725.2004.30431.x.

5. Schwarz T, von Hodenberg E, Furtwängler C, Rastan A, Zeller T, Neumann FJ. Endovenous laser ablation of varicose veins with the 1470-nm diode laser. J Vasc Surg. 2010;51:1474-1478. doi:10.1016/j.jvs.2010.01.027.

6. Vuylsteke ME, Vandekerckhove PJ, De Bo T, Moons P, Mordon S. Use of a new endovenous laser device: results of the 1,500 nm laser. Ann Vasc Surg. 2010;24:205-211. doi:10.1016/j.avsg.2009.06.024.

7. Vuylsteke ME, Thomis S, Mahieu P, Mordon S, Fourneau I. Endovenous laser ablation of the great saphenous vein using a bare fibre versus a tulip fibre: a randomised clinical trial. Eur J Vasc Endovasc Surg. 2012;44:587-592. doi:10.1016/j.ejvs.2012.09.003.

8. Weiss RA, Weiss MA. Controlled radiofrequency endovenous occlusion using a unique radiofrequency catheter under duplex guidance to eliminate saphenous varicose vein reflux: a 2 -year follow-up. Dermatol Surg.
2002;28:38-42. doi:10.1097/00042728-200201000-00009.

9. Lurie F, Creton D, Eklof B, et al. Prospective randomized study of endovenous radiofrequency obliteration (closure procedure) versus ligation and stripping in a selected patient population (EVOLVeS Study). J Vasc Surg. 2003;38:207-214. doi:10.1016/s0741-5214(03)00228-3.

10. Park JY, Galimzahn A, Park HS, Yoo YS, Lee T. Midterm results of radiofrequency ablation for incompetent small saphenous vein in terms of recanalization and sural neuritis. Dermatol Surg. 2014;40:383-389. doi:10.1111/ dsu. 12456.

11. Badham GE, Strong SM, Whiteley MS. An in vitro study to optimise treatment of varicose veins with radiofrequencyinduced thermo therapy. Phlebology. 2015;30:17-23. doi: $10.1177 / 0268355514552005$.

12. Almeida JI, Kaufman J, Göckeritz O, et al. Radiofrequency endovenous ClosureFAST versus laser ablation for the treatment of great saphenous reflux: a multicenter, single-blinded, randomized study (RECOVERY study). J Vasc Interv Radiol. 2009;20:752-759. doi:10.1016/j. jvir.2009.03.008.

13. Rasmussen LH, Lawaetz M, Bjoern L, Vennits B, Blemings A, Eklof B. Randomized clinical trial comparing endovenous laser ablation, radiofrequency ablation, foam sclerotherapy and surgical stripping for great saphenous varicose veins. Br J Surg. 2011;98:1079-1087. doi:10.1002/ bjs.7555.

14. Doganci S, Demirkilic U. Comparison of $980 \mathrm{~nm}$ laser and bare-tip fibre with $1470 \mathrm{~nm}$ laser and radial fibre in the treatment of great saphenous vein varicosities: a prospective randomised clinical trial. Eur J Vasc Endovasc Surg. 2010;40:254-259. doi:10.1016/j.ejvs.2010.04.006.

15. Evans CJ, Fowkes FG, Ruckley CV. Prevalence of varicose veins and chronic venous insufficiency in men and women in the general population: Edinburgh Vein Study. J Epidemiol Community Health. 1999;53:149-153. doi:10.1136/jech.53.3.149.

16. Almeida JI, Raines JK. Radiofrequency ablation and laser ablation in the treatment of varicose veins. Ann Vasc Surg. 2006;20:547-752. doi:10.1007/s10016-006-9098-8.

17. Puggioni A, Kalra M, Carmo M, Mozes G, Gloviczki P. Endovenous laser therapy and radiofrequency ablation of the great saphenous vein: analysis of early efficacy and complications. J Vasc Surg. 2005;42:488-493. doi:10.1016/j. jvs.2005.05.014.

18. Gale SS, Lee JN, Walsh ME, Wojnarowski DL, Comerota AJ. A randomized, controlled trial of endovenous thermal ablation using the 810 -nm wavelength laser and the ClosurePLUS radiofrequency ablation methods for superficial venous insufficiency of the great saphenous vein. J Vasc Surg. 2010;52:645-650. doi:10.1016/j.jvs.2010.04.030.

19. Shepherd AC, Gohel MS, Brown LC, Metcalfe MJ, Hamish M, Davies AH. Randomized clinical trial of VNUS ClosureFAST radiofrequency ablation versus laser for varicose veins. Br J Surg. 2010;97:810-818.

20. Shepherd AC, Gohel MS, Lim CS, Hamish M, Davies AH. Pain following 980-nm endovenous laser ablation and segmental radiofrequency ablation for varicose veins: a prospective observational study. Vasc Endovascular Surg. 2010;44:212-216. doi:10.1177/1538574409359337.

21. Van den Bos R, Arends L, Kockaert M, Neumann M, Nijsten T. Endovenous therapies of lower extremity varicosities: a meta-analysis. J Vasc Surg. 2009;49:230-239. 